\title{
Dynamic single-molecule sensing via nanoparticle micromanipulation for rapid and ultrasensitive biomarker detection
}

\section{Qiang Zeng}

Shanghai Jiao Tong University

\section{Xiaoyan Zhou}

Shanghai Jiao Tong University

\section{Yuting Yang}

Shanghai Jiao Tong University

Yi Sun

Tsinghua University

Jingan Wang

Shanghai Jiao Tong University

Chunhui Zhai

Shanghai Jiao Tong University

Jinghong Li

Tsinghua University https://orcid.org/0000-0002-0750-7352

Hui Yu ( $\nabla$ hui.yu@sjtu.edu.cn )

Shanghai Jiao Tong University https://orcid.org/0000-0003-4430-2230

\section{Article}

Keywords: Sensing Single Molecule under MicroManipulation (SSM3), single-molecule sensors, biomarker detection

Posted Date: October 8th, 2021

DOl: https://doi.org/10.21203/rs.3.rs-950977/v1

License: (c) (i) This work is licensed under a Creative Commons Attribution 4.0 International License. Read Full License 


\section{Dynamic single-molecule sensing via nanoparticle micromanipulation for rapid and ultrasensitive biomarker detection}

Qiang Zeng ${ }^{1 \#}$, Xiaoyan Zhou $^{1 \#}$, Yuting Yang $^{2 \#}$, Yi Sun $^{3}$, Jingan Wang ${ }^{1}$, Chunhui Zhai $^{1}$, Jinghong $\mathrm{Li}^{3^{*}}$, Hui $\mathrm{Yu}^{1,4^{*}}$

${ }^{1}$ School of Biomedical Engineering, Shanghai Jiao Tong University, Shanghai, 200030, People's Republic of China

${ }^{2}$ Department of Instrument Science and Engineering, School of Electronic Information and Electrical Engineering, Shanghai Jiao Tong University, Shanghai, 200030, People's Republic of China

${ }^{3}$ Department of Chemistry, Key Laboratory of Bioorganic Phosphorus Chemistry \& Chemical Biology, Tsinghua University, Beijing, 100084, People's Republic of China

${ }^{4}$ Institute of Medical Robotics, Shanghai Jiao Tong University, Shanghai, 200030, People's Republic of China

"These authors contributed equally to this work.

${ }^{*}$ Correspondence should be addressed to: H.Y. (hui.yu@sjtu.edu.cn) or J.L. (jhli@mail.tsinghua.edu.cn) 


\section{Abstract}

The ability to measure many single molecules simultaneously in larger and complex samples is critical to the translation of single-molecule sensors for practical applications in biomarker detection. The challenges lie in the limits imposed by mass transportation and thermodynamics, resulting in long assay time and/or insufficient sensitivity. Here, we report an approach called Sensing Single Molecule under MicroManipulation $\left(\mathrm{SSM}^{3}\right)$ to circumvent the above limits. In $\mathrm{SSM}^{3}$, the transportation rate of analyte molecules and the kinetics of molecular interaction are fine-tuned by the nanoparticle micromanipulation. The heterogeneous lifetime of molecular complexes is quantified to discriminate specific binding from nonspecific background noise. By the highly-specific digital counting of single molecules, we demonstrate 15-minute assays for direct detection of microRNAs and amyloid- $\beta$ proteins via electrical or magnetic micromanipulation, with the limit of detection at the subfemtomolar level. The presented approach could inspire more practical applications of single molecule sensors. 
The analytical methods have converged from ensemble measurements of numerous entities to quantized measurements at the single-molecule level. Single-molecule measurements could reveal heterogeneities and stochastic processes within biological systems ${ }^{1,2}$, and set the ultimate detection limit of chemical and biological sensors. By reducing the measurement volume to a few femtoliters, the detection of a single molecule has been realized in various forms, i.e. single molecule fluorescence ${ }^{3,4}$, nanopores ${ }^{5,6}$, localized surface plasmon resonance ${ }^{7,8}$, and surface enhanced Raman scattering ${ }^{9,}{ }^{10}$. These measurements typically require quantifying many single-molecule events to gain new molecular and mechanistic insights or to achieve better analytical performance. However, it has been difficult to perform quantitative analysis with sufficient efficiency and statistical accuracy, due to the concentration limit from mass transportation $^{11,12}$ and the thermodynamic limit from probe affinity ${ }^{13}$. For quantification of biomarkers in biological media, where the required concentrations are usually at the femtomolar level or even lower ${ }^{14}$, the single-molecule measurements could take inordinately long, and the nonspecific binding of unwanted species degrades the accuracy.

In the past two decades, several single-molecule approaches for biomarker detection have been developed to surpass the above limits by biasing the equilibrium and driving binding reactions ${ }^{15,16}$. A typical scheme involves the usage of nanoparticles to collect the analyte followed by a digital measurement of single molecules at a confined space ${ }^{17}$, such as the commercialized single-molecule enzyme-linked immunosorbent analysis (digital ELISA) ${ }^{18}$. The digital ELISA uses the antibody-modified magnetic beads to capture the analyte in solution, and loads them into femtoliter-sized reaction chambers termed single-molecule arrays (SiMoA). It effectively improves the sensitivity of conventional ELISA by 3 orders with a limit of detection at 
the subfemtomolar level, but requires sophisticated devices and excessive operation to remove free analyte molecules. Besides, the performance is still limited by the probe affinity and false positive can arise from detection antibodies that bind nonspecifically to assay surface.

A distinct yet effective strategy is by exploring the in-depth heterogeneous information of single-molecule interaction ${ }^{19}$. Walter et al. first demonstrated a kinetic fingerprinting approach to perform highly specific and sensitive detection of biomarkers via single-molecule fluorescence microscopy ${ }^{20,21,22}$. This single-molecule recognition through equilibrium Poisson sampling (SiMREPS) technique surpasses the thermodynamic limit by exploiting the repetitive binding of fluorescently labeled low affinity probes to the analyte ${ }^{23}$, and discriminates specific binding from background noise by a kinetic signature. The detection limits of miRNAs and proteins also reach the subfemtomolar level, but screening probes with unique kinetic property is costly and laborious, and the concentration limit implies long incubation time before detection.

Herein, we present the integration of single-molecule manipulation and dynamic sensing to allow rapid and ultrasensitive detection of biomarkers beyond the concentration and thermodynamic limits. In this Sensing Single Molecule under MicroManipulation $\left(\mathrm{SSM}^{3}\right)$ approach, an external force is applied on the molecular bound between analyte and probes through tethered nanoparticles to actively tune the binding kinetics. This strategy, together with a dynamic sensing approach to exploit the heterogeneity at the single-molecule level, is able to beat the limits in both assay time and sensitivity. We show the principle and realization of the $\mathrm{SSM}^{3}$ technique, and demonstrate 15-minute assays to directly measure microRNAs (miRNAs) and proteins at the subfemtomolar concentration. 


\section{The principle}

The single-molecule detection was performed in a typical sandwich scheme composed of a high-affinity primary probe immobilized on a planar sensor chip, the analyte and a secondary probe tethered to nanoparticles (Figure 1a, and supplementary information). A microscope with single nanoparticle imaging capability, such as the surface plasmon resonance microscope (SPRM) used herein, dynamically records the image sequences of nanoparticles to report single molecular interactions ${ }^{24,25,26,27}$. Considering the first-order binding kinetics between the analyte and the secondary probe, $\mathrm{SSM}^{3}$ reports the sum of total association and dissociation events during the observation by digital counting at the single-molecule level (Eq.1),

$$
N(\tau)=\int_{0}^{\tau}\left(k_{\mathrm{on}}[\mathrm{A}]_{t}[\mathrm{P}]_{t}+k_{\mathrm{off}}[\mathrm{AP}]_{t}\right) \mathrm{d} t
$$

where $k_{\text {on }}$ and $k_{\text {off }}$ are the association and dissociation rate constants, $[\mathrm{A}],[\mathrm{P}]$ and $[\mathrm{AP}]$ are the concentration of free analyte, free probes and the analyte-probe complexes. This readout increases continuously with longer observation time even after reaching equilibrium, so that the detection limit is only determined by the background noise from nonspecific binding. This is obviously different from the conventional endpoint ensemble measurement where the limited binding affinity sets the maximum readout (Supplementary information).

The $\mathrm{SSM}^{3}$ also reports the duration of each binding event, which is determined by the association and dissociation kinetics ${ }^{28}$. As a result, we could count only the specific binding in Eq.1, and obtain an ultrahigh sensitivity beyond the thermodynamic limit. But it is critical to use an appropriate low-affinity probe with rapid binding and dissociation kinetics, so that enough 
number of dissociation events could be identified for statistical analysis in limited assay time. $\mathrm{SSM}^{3}$ makes use of the single-molecule manipulation ${ }^{29,30}$ to fine-tune the binding kinetics of commonly used high-affinity probes. Considering the one-dimensional system (Figure 1b), the thermodynamics could be described by the Langevin equation, ${ }^{31}$

$$
M \frac{\mathrm{d}^{2} z}{d t^{2}}=-\frac{\mathrm{d} U(z)}{\mathrm{d} z}-6 \pi \eta a \frac{d z}{d t}+\xi(t)
$$

where $M$ is the mass of the nanoparticle, $U(z)$ is the restoring potential related to the molecular binding energy, the second term on the right describes the damping due to viscosity ( $\eta$ is the solvent viscosity and $a$ is the radius of the nanoparticle), and $\xi$ is the thermal fluctuation force. The probability $\rho(\mathrm{t})$ that a molecule persists in its bound state can be approximately calculated through the kinetic equation as defined by Kramer's transition rate ${ }^{32}$

$$
\frac{\mathrm{d} \rho(t)}{\mathrm{d} t}=-\frac{\omega_{0} \omega_{1} M}{12 \pi^{2} \eta a} e^{-\left(U_{B}^{\prime}(t) / k_{B} T\right)} \rho(t)
$$

where $U_{B}^{\prime}$ is the barrier height, $\omega_{0}$ and $\omega_{1}$ are the effective oscillation frequencies at the bound state and at the maximum potential, $k_{\mathrm{B}}$ is Boltzmann constant, and $T$ is the temperature. The Monte-Carlo simulation shows that when lowering the energy barrier with an external force (Figure 1c), the distribution shifts towards the shorter timescale as expected and consequently the frequency of repetitive binding increases (Figure 1d). 
a Micromanipulation \& Imaging

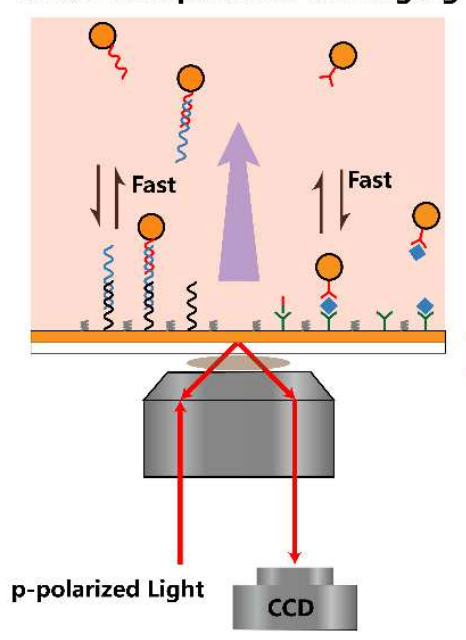

b

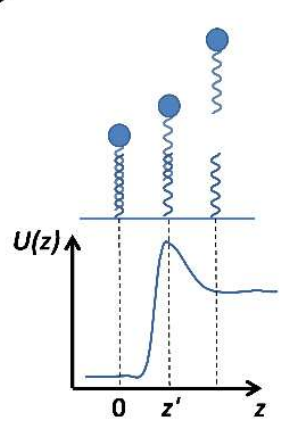

Single particle analysis

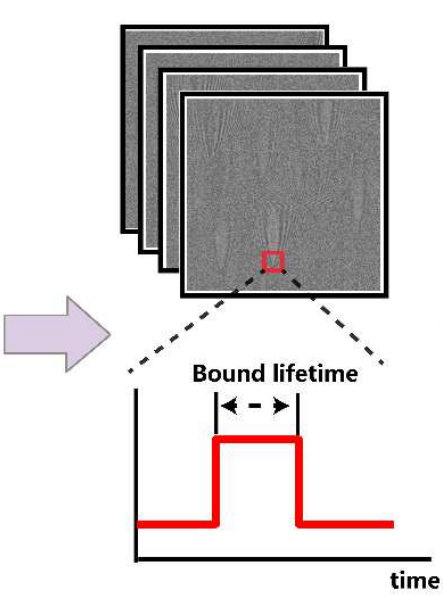

Digital sensing

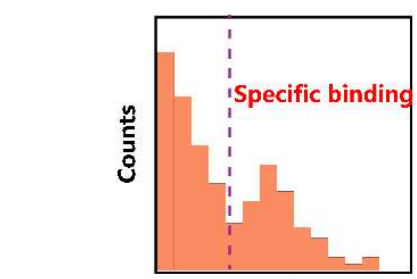

Bound lifetime (s)

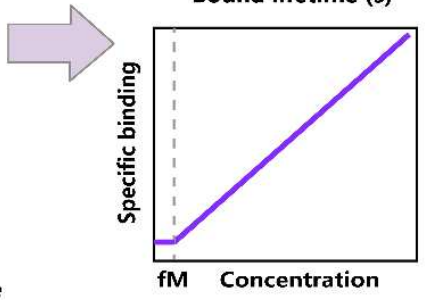

d
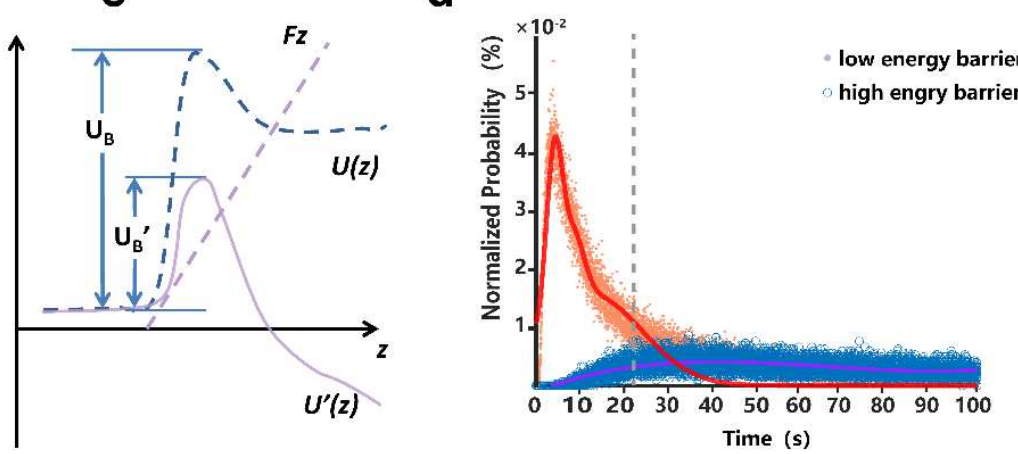

Figure 1 The principle of $\mathrm{SSM}^{3}$. (a) The schematics; (b) the one-dimensional model system; (c) the free energy level; (d) the distribution of bound lifetime under different energy barriers by the Monte-Carlo simulation.

\section{The effectiveness of micromanipulation}

To prove the effectiveness of the nanoparticle micromanipulation and dynamic sensing strategy,

we first explored the miRNA detection by electrical manipulation of $50-\mathrm{nm}$ Au nanoparticles (AuNPs), owing to the compatibility of SPRM with electrochemical systems (Figure 2a). ${ }^{33,34}$ DNA probes with 12 nucleotides complementary to the target miRNA hsa-miR-29a were tethered to the Au nanoparticles, while high-affinity locked nucleic acid (LNA) probes were immobilized on the planar Au sensor surface as the capture probes. With the concentration of AuNPs at $10^{10} / \mathrm{mL}$ ( $16 \mathrm{pM})$, the chance of more than one analyte molecule binding to a same AuNP is less than $1.8 \%$ 
at an analyte concentration below $3.2 \mathrm{pM}$ according to the Poisson statistics ${ }^{35}$. When examining the distribution of bound lifetime, the specific binding to hsa-miR-29a could be discriminated from nonspecific binding (Figure $\mathbf{2 b}$ ). This was confirmed by the nonspecific binding in the control group without analyte, where most nanoparticles hit and run quickly, and a very small portion was absorbed on surface firmly. (Figure S1, Supplementary information).

The rising peak in the simulation results of Figure $1 \mathbf{d}$ was overwhelmed by the large number of nonspecific binding. We thus empirically set a threshold of the lifetime at $25.6 \mathrm{~s}$, within which $95 \%$ of the nonspecific binding in the control group dissociated. The lifetime of specific binding over the threshold was fitted by an exponential function to find the mean lifetime, which reflects the potential barrier height as in Eq. 3. Without applying a voltage, the mean lifetime of molecular complexes was found to be $35.04 \pm 1.21 \mathrm{~s}$ (Figure $2 \mathrm{c}$ ), which corresponds well to the expectation for probes with a typical dissociation constant in the micromole-to-nanomole range ${ }^{28}$. With a negative voltage applied, the AuNPs (zeta potential: $-31.67 \mathrm{mV}$ ) were pushed away from the surface. The mean lifetime thus decreased and the readout in Eq.1 increased. Note that when the energy barrier diminishes at a higher negative voltage, it will largely impede the binding process and the readout did not further increase. Empirically, the optimal manipulation was found at $0.2 \mathrm{~V}$, where the mean lifetime decreased to $29.64 \pm 0.65 \mathrm{~s}$, and the readout increased by up to 3 folds.

The discrimination of specific binding by the lifetime of molecular complex leads to improved limit of detection (LOD). The LoD by direct counting of all binding events within the 0-300 s lifetime window was $14.88 \mathrm{pM}$, which is similar to the previously reported digital assay ${ }^{24,36}$. 
When counting only specific binding within the $25-250 \mathrm{~s}$ window, the LoD was found to be 0.36

fM with a high linearity (Figure $\mathbf{2 d}$ ). Although a large number of events were rejected, most of them were due to the nonspecific bindings as could be seen from the significant lowering of baseline (dashed lines). When examining the results within $0-25 \mathrm{~s}$, it shows a poor linearity as the nonspecific binding dominates. These results thus prove the effectiveness of using in-depth heterogeneous information at the single-molecule level to improve the sensing performances

a

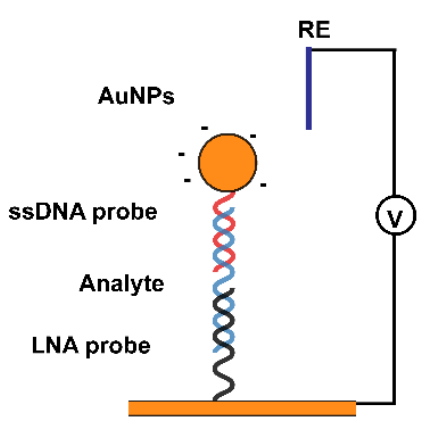

C

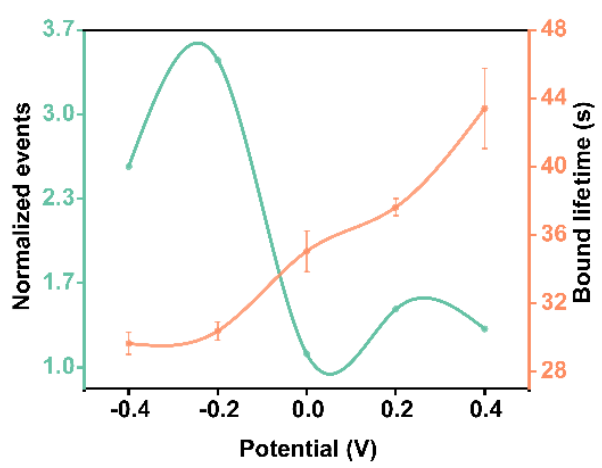

b

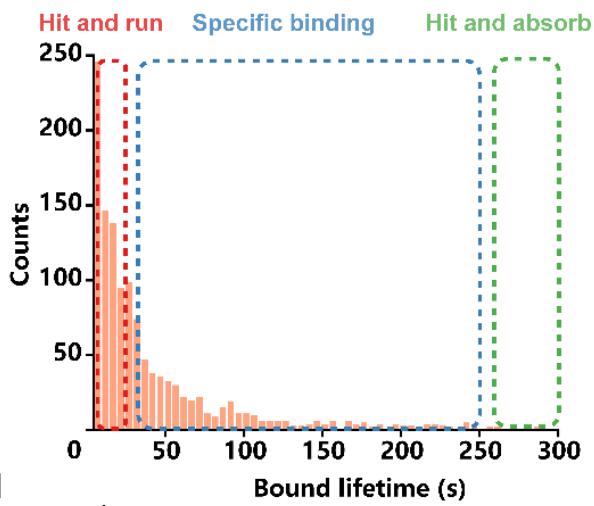

d

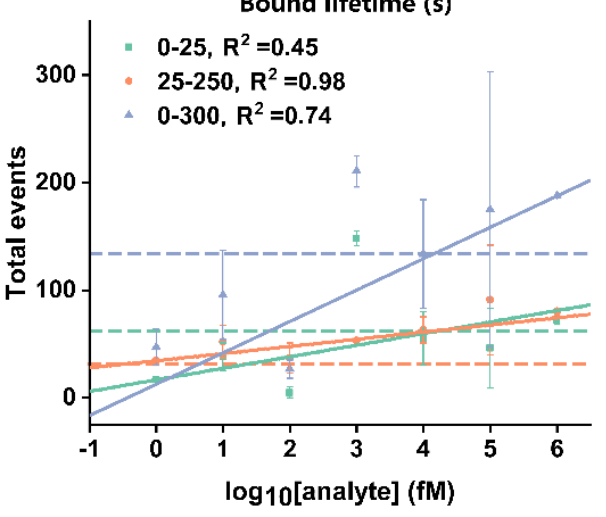

Figure 2 Effect of nanoparticle manipulation on the single molecule sensing performance. (a) The $\mathrm{SSM}^{3}$ system with electrical manipulation; (b) the lifetime distribution of molecular bound; (c) the mean lifetime and readout signal under different applied voltages; (d) the concentration dependent sensing performance from different lifetime windows.

\section{Rapid miRNA testing assay}

We then demonstrate the capability of $\mathrm{SSM}^{3}$ in developing the 15 -minute assay to detect miRNAs 
(Figure 3a). At a low concentration, the pre-incubation of analyte on the coated planar sensor could take tens of minutes, limiting the full assay time. Instead, the analyte was mixed with the probe-coated Au nanoparticles, and immediately injected to the testing chamber without further incubation. A voltage was applied sequentially as $+0.4 \mathrm{~V}$ for 5 minutes to facilitate transportation of analyte and AuNPs onto the sensor surface, followed by $-0.2 \mathrm{~V}$ for 10 minutes to promote repetitive binding. The image sequences were recorded immediately after injection and continuously throughout the assay. Within 15 minutes, the time-dependent coefficient of variation (CV) decreased to below $15 \%$, indicating that the assay time is enough to reach a good stability in readout (Figure S3, Supplementary information).

To prove the effectiveness and generality, we tested three synthetic disease-related miRNAs including hsa-miR-21-5p (miR-21), hsa-miR-155-5p (miR-155) and hsa-miR-362-5p (miR-362) (Supplementary information). Despite varied binding kinetics, the lifetime distribution of specific binding could be distinguished from unspecific binding by setting the threshold at $5 \mathrm{~s}$ for all three miRNAs (Figure S2, Supplementary information). The LoD for miR-21, miR-155 and miR-362 were found to be $0.26 \mathrm{fM}, 0.17 \mathrm{fM}$ and $0.15 \mathrm{fM}$ respectively, with linear detection ranges over 6 orders of magnitude (Figure $\mathbf{3 b}$ ). In the receiver operating characteristic (ROC) plot, the area under curve (AUC) values were $0.997,0.988$ and 0.967 respectively (Figure $3 c$ ). In principle, a more rigorous threshold setting could further improve the specificity, but at the cost of less countable events and consequently longer assay time.

The 15-minute $\mathrm{SSM}^{3}$ assay could also be used to discriminate single-base mismatch, owing to its high specificity. Two diseases-related miRNAs, the hsa-miR-29a and hsa-miR-29c, were 
synthesized with a single nucleotide difference (Supplementary information), and the detection probe was 12-nt complimentary to hsa-miR-29a. The mean lifetime of hsa-miR-29a and hsa-miR-29c were found to be $21.47 \pm 0.45 \mathrm{~s}$ and $10.42 \pm 0.96 \mathrm{~s}$ respectively (Figure $\mathbf{3 d}$ ). We thus empirically set a threshold at $14 \mathrm{~s}$, and found that the tests only show dependency on the concentration of hsa-miR-29a (Figure 3e). The distinct sensing performance guarantees high specificity with a discrimination factor over 141 for detection of $h s a-m i R-29 a$ and $h s a-m i R-29 c$. In the presence of hsa-miR-29c at 100-fold higher concentrations, the LoD of hsa-miR-29a was found to be $0.63 \mathrm{fM}$ with an AUC of 0.816 (Figure S4, Supplementary information). The detection of hsa-miR-29a with or without the existence of hsa-miR-29c further verified the effectiveness (slope $=0.92, R>0.99$ ) (Figure 3f).
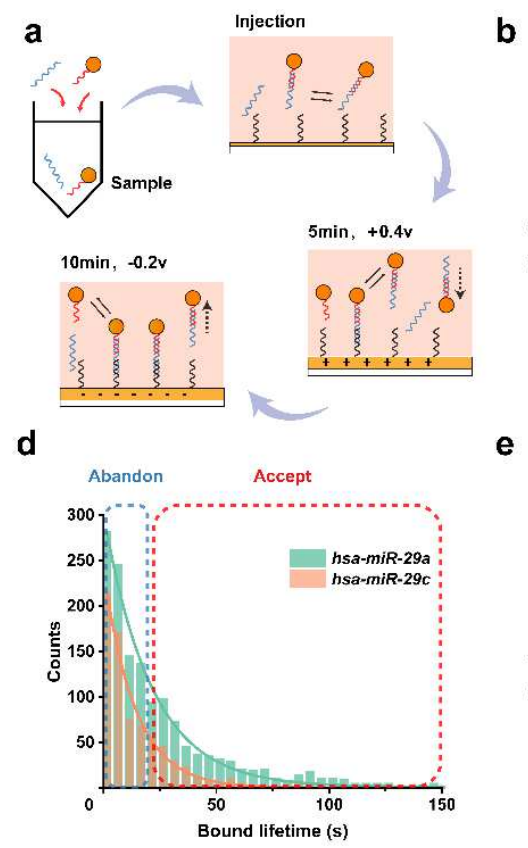

b
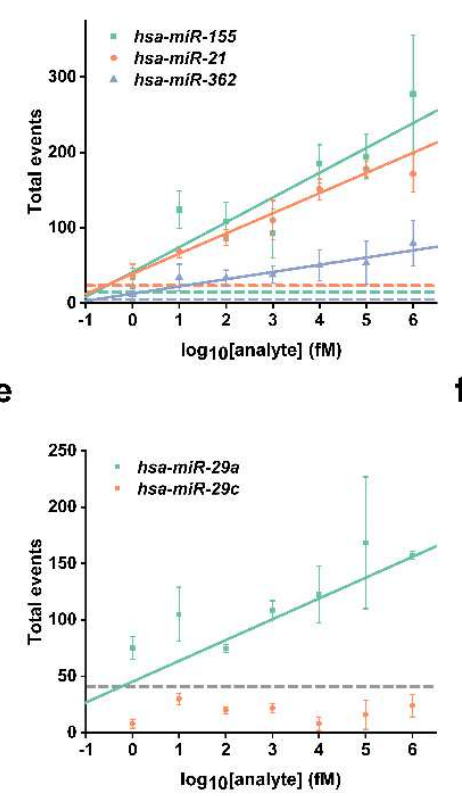

c

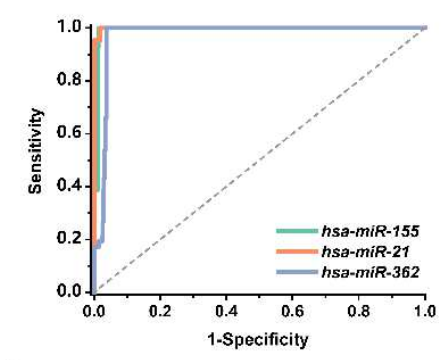

f

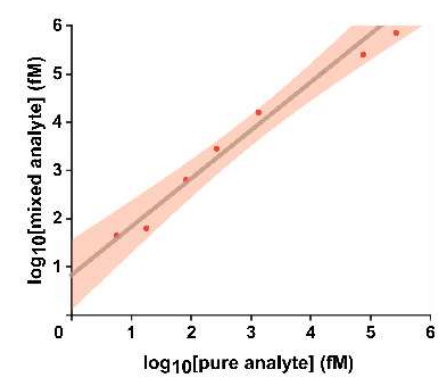

Figure 3 Rapid miRNA testing assay. (a) The workflow of 15-minute miRNA assay; (b) the calibration curve and (c) the ROC plot for hsa-miR-21-5p, hsa-miR-155-5p and hsa-miR-362-5p detection; (d) lifetime distribution of molecular bound between the probes and hsa-miR-29a or hsa-miR-29c; (e) calibration curve for hsa-miR-29a and hsa-miR-29c detection; (f) measured concentration of the hsa-miR-29a with or without the existence of hsa-miR-29c. 


\section{$\mathrm{SSM}^{3}$ immunoassay}

The $\mathrm{SSM}^{3}$ is also compatible with the immunoassay for protein detection, by replacing the DNA probes with corresponding antibodies. We then demonstrate the ultrasensitive detection of beta amyloid protein $42\left(A \beta_{1-42}\right)$, an important molecular biomarker for neurodegenerative disease diagnostics in body fluid. Primary antibodies were coated on the planar sensor surface and on AuNPs in the sandwich system (Figure 4a). Under optimized condition, the electrical manipulation shortened the mean lifetime for protein detection from $45.05 \pm 1.36 \mathrm{~s}$ to $37.38 \pm$ $1.44 \mathrm{~s}$ (Figure 4b), and the LoD for $A \beta_{1-42}$ detection reached $4.34 \mathrm{fg} / \mathrm{mL}(\sim 1.08 \mathrm{fM}$ ) (Figure $4 \mathrm{c}$ ). Note that the mean lifetime of $\sim 45 \mathrm{~s}$ in immunoassay is longer than $\sim 35 \mathrm{~s}$ in miRNA assay due to the higher affinity between protein and antibody interaction, imposing the requirement in a higher applied force.

To further improve the performance, we introduced the magnetic manipulation scheme, which has been used in magnetic tweezers to provide the pico-Newton level force to break down ligand-receptor complexes ${ }^{37}$. The magnetic manipulation involves the use of 300-nm magnetic nanoparticles and external magnetic fields to replace the AuNPs and electric fields (Figure 4a). The binding kinetics between $A \beta_{1-42}$ proteins and antibodies was adjusted in a wider range by changing the distance between the magnets and the sensor surface, reducing the bound lifetime to $22.22 \pm 2.54 \mathrm{~s}$ (Figure 4b). At optimal experimental conditions, the LoD via magnetic manipulation was found to be $0.21 \mathrm{fg} / \mathrm{mL}(\sim 0.05 \mathrm{fM})$, which is 20 times better than that via electrical manipulation (Figure 4c). 
a

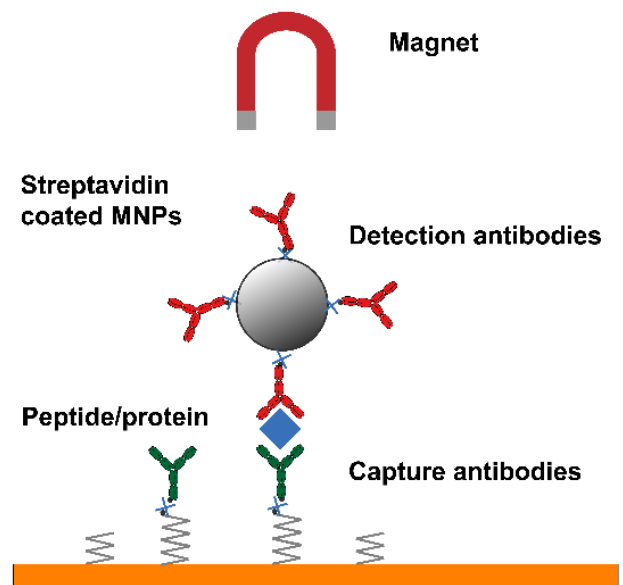

b
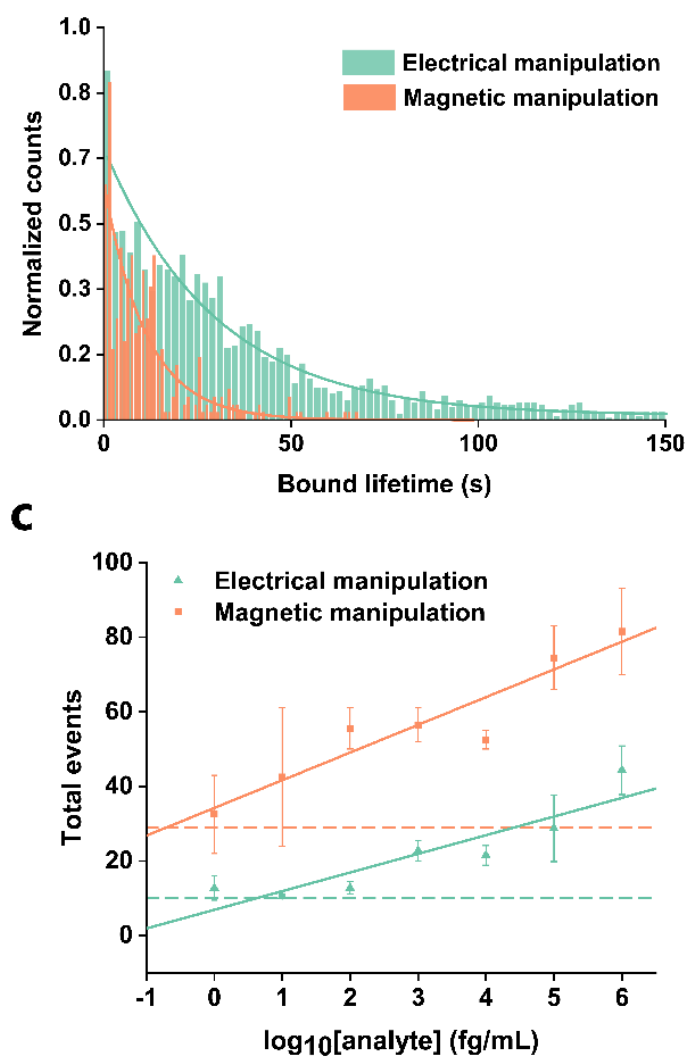

Figure 4 Rapid immunoassay for $A \beta_{1-42}$ protein detection. (a) The schematics of immunoassay via magnetic manipulation; (b) the bound lifetime distribution and (c) the calibration curve via electrical and magnetic manipulation.

\section{Spiked miRNA and protein test in human serum}

To further verify the effectiveness of $\mathrm{SSM}^{3}$ assay in complex samples, we performed the 15-minute assay to detect $h s a-m i R-29 a$ and $A \beta_{1-42}$ proteins spiked in human serum collected from 
healthy volunteers. Under electrical manipulation, the LoD for spiked hsa-miR-29a detection was $1.51 \mathrm{fM}$ (Figure 5a). As the $A \beta_{1-42}$ protein level in human serum is usually on the level of $\mathrm{pg} / \mathrm{mL}^{38}$, we pretreated the serum by proteinase $\mathrm{K}$ to digest existing proteins, followed by a $90{ }^{\circ} \mathrm{C}$ treatment to further inactivate remaining proteins. The LoDs for spiked $A \beta_{1-42}$ protein detection in the pretreated human serum were $21.87 \mathrm{fg} / \mathrm{mL}(4.84 \mathrm{fM})$ via electrical manipulation and 6.76 $\mathrm{fg} / \mathrm{mL}(1.49 \mathrm{fM})$ via magnetic manipulation (Figure 5b). With this calibration curve, we then directly measured the $A \beta_{1-42}$ protein level in untreated human serum, yielding the concentration of $18.93 \pm 3.73 \mathrm{pg} / \mathrm{mL}$ that is within the typical range reported ${ }^{38,39}$.

\section{Discussion}

We have presented the Sensing Single Molecule under MicroManipulation $\left(\mathrm{SSM}^{3}\right)$ for ultrasensitive detection of molecular biomarkers with analytical performance beyond the concentration and thermodynamic limits. Instead of screening for unique low-affinity probes in the kinetic fingerprinting approaches, the electrical and magnetic manipulation of nanoparticles allows for fine tuning of molecular binding kinetics of high-affinity probes. In the 15-minute assay, the LoD for detecting miRNAs reaches the subfemtomolar level, and single base pair mismatch could be identified with a discrimination factor over 140 . For $A \beta_{1-42}$ protein detection, the LoD reaches $0.21 \mathrm{fg} / \mathrm{mL}$, three orders of magnitude higher than conventional immunoassay. The preliminary study using spiked human serum implies its effectiveness in complex sample detection. Compared with the commercialized digital ELISA technique, the $\mathrm{SSM}^{3}$ is superior in terms of shorter assay time, lower cost and simpler workflow, benefiting from the usage of heterogeneous single-molecule information. We anticipate that further improvements on the 
multiplexing capability, imaging throughput and finer manipulation could largely advance the wide applications in fundamental biological research and clinical detections.

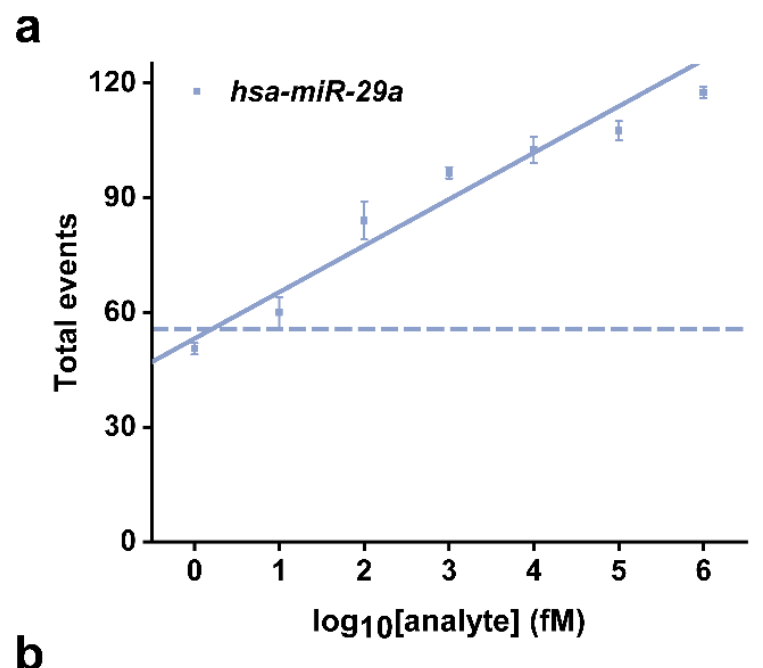

b

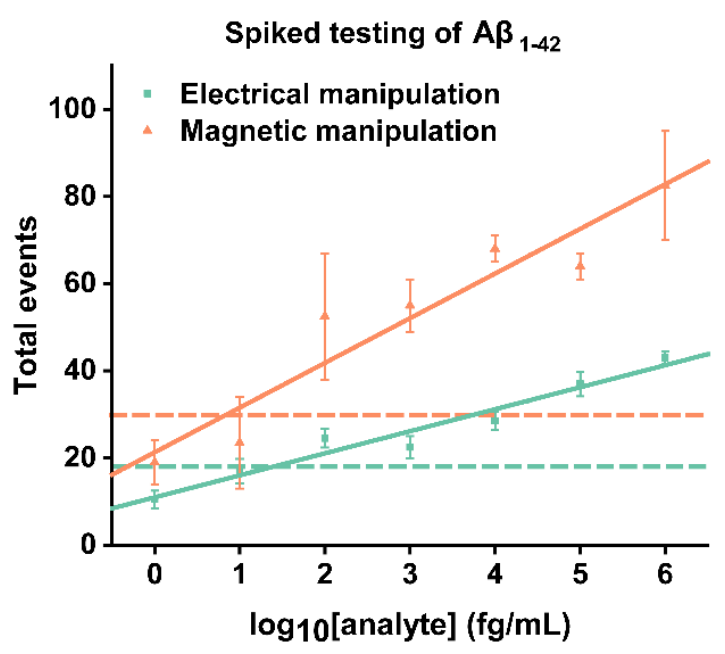

Figure 5 Detection of miRNAs and proteins spiked in human serum. (a) The calibration curve for spiked hsa-miR-29a detection in human serum via electrical manipulation; (b) the calibration curve for spiked $A \beta_{1-42}$ protein detection in pretreated human serum via electrical and magnetic manipulation.

\section{Methods}

Materials. All oligonucleotides used in this work were synthesized by Sangon Biotech (Shanghai) Co., Ltd. Detailed sequences are listed in the Supplementary information. Phosphate buffered 
saline (PBS) buffer, DEPC-treated water (diethyl pyrocarbonate) and TE (Tris-EDTA) buffer were purchased from Sangon Biotech (Shanghai) Co., Ltd. The streptavidin-functionalized magnetic nanoparticle (MNP), Tris-(2-carboxyethyl)-phosphine hydrochloride (TCEP) and Sodium dodecyl sulfate (SDS) were purchased from Aladdin. Proteinase $\mathrm{K}$ and Tris-HCL buffer were purchased from Beyotime Biotechnology. $A \beta_{1-42}$ peptide and monoclonal antibodies (mAbs) of $A \beta_{1-42}$ (clone 12F4) specific to the $\mathrm{C}$-termini, and a mAb capable of binding to the $\mathrm{N}$-terminus of $A \beta_{1-42}$ peptide (clone 6E10) were obtained from BioLegend, Inc. The streptavidin-functionalized gold nanoparticles (AuNPs) were purchased from Nanopartz. Thiol-PEG-hydroxyl ( $M w=500$ Da) Thiol-PEG-biotin ( $\mathrm{Mw}=3400 \mathrm{Da}$ ) and streptavidin was purchased from Sigma-Aldrich. All solutions were prepared with deionized water $\left(18.2 \mathrm{M} \Omega \mathrm{cm}^{-1}\right)$ from a Millipore system. All experiments were repeated in triplicates unless mentioned otherwise.

Surface plasmon resonance microscopy. The $\mathrm{SSM}^{3}$ experiments were performed using a previously described SPRM system ${ }^{26,27}$. Briefly, the SPRM was built on an inverted total internal reflection microscope (IX83, Olympus, Japan) equipped with a $60 \times$ oil-immersion objective. Collimated light from a superluminescent diode (SLD635B, Thorlabs) illuminated the 50-nm Au on BK-7 glass sensor chip at the resonant angle. Images were acquired by a SCMOS camera (Photometrics Prime 95B) with a full field of view of $102.4 \times 102.4 \mu \mathrm{m}^{2}$ at a frame rate of 16.7 fps. The image sequences were processed during acquisition by a home-developed software package $^{40}$.

Sandwich structure. For miRNA detection, the $\mathrm{SSM}^{3}$ exploited the direct binding of a long detection-probe-labeled AuNPs to an unlabeled oligo-analyte captured on a gold film. The surface of gold-coated glass slide was passivated with $20 \mu \mathrm{L}$ of Thiol-PEG-hydroxyl spacer at a 
concentration of $1 \mu \mathrm{M}$ in $1 x$ PBS for 30 seconds. Then the slide was rinsed three times with $1 x$ PBS and incubated with $20 \mu \mathrm{L}$ of $50 \mathrm{nM}$ thiol-ssDNA capture probes in $1 \times$ PBS solution for $2 \mathrm{~h}$. The capture probe was pretreated with $1 \mathrm{mM}$ TCEP to reduce the thiol groups and activate the thiolated DNA. The surface was finally passivated by adding $20 \mu \mathrm{L}$ of the spacer solution and incubated for $20 \mathrm{~min}$, and rinsed with 1x PBS three times to remove the unbound molecules. As for protein sensing, the ssDNA capture probe was replaced by the biotinylated antibody. In brief, the planar sensor surface was first modified with Thiol-PEG-biotin at the concentration of $1 \mu \mathrm{M}$ in 1x PBS for $15 \mathrm{~min}$, followed by incubation in $1 \mathrm{mg} / \mathrm{mL}$ streptavidin in Tris-HCL buffer for $1 \mathrm{~h}$. mAb probe (clone 6E10) at a concentration of $5 \mathrm{ug} / \mathrm{mL}$ was added and incubated for $2 \mathrm{~h}$.

The 50nm streptavidin-coated AuNP (C11-50-TS-PBS-50-1) at a concentration of $10^{10} \mathrm{NPs} / \mathrm{mL}$ was incubated with $100 \mu \mathrm{L}$ of biotinylated DNA detection probe or biotinylated antibody (clone 12F4) at a concentration of $1 \mu \mathrm{M}$ in $3 \mathrm{mM}$ PBS for $30 \mathrm{~min}$. To note, $40 \times$ diluted PBS with an ionic concentration of $3 \mathrm{mM}$ was used to prevent the gold nanoparticle from aggregation. For miRNA detection, the detection probe has the adjustable complementary length to oligo-analyte. AuNPs were rinsed by $3 \mathrm{mM}$ PBS three times to remove the redundant DNA, and diluted to $10^{10} \mathrm{NPs} / \mathrm{mL}$. For magnetic manipulation, the $300 \mathrm{~nm}$ streptavidin-coated MNP (S8040-A300nm-1EA) at a concentration of $10^{9} \mathrm{NPs} / \mathrm{mL}$ was incubated with biotinylated antibody (clone 12F4) for $2 \mathrm{~h}$, at a final concentration of $2.5 \mathrm{ug} / \mathrm{mL}$ in Tris-HCL buffer. The mAb-coated magnetic nanoparticles were centrifuged and re-suspended for three time to remove the redundant reagent.

Micromanipulation system. The electrical micromanipulation system was built with an electrochemical workstation ( $\mathrm{CHI660e}, \mathrm{CH}$ Instruments Inc.). The counter-electrode (CHI115 platinum wire) and reference electrode $(\mathrm{CHI} 111 \mathrm{Ag} / \mathrm{AgCl})$ were placed in a silicon solution cell 
(flexi-PERM, SARSTEDT), and the Au film served as the working electrode. The voltage is applied between working electrode and reference electrode. For verification of the effect in tuning binding kinetics, the voltage was scanned from $-0.4 \mathrm{~V}$ to $+0.4 \mathrm{~V}$. For magnetic micromanipulation, a magnet was placed above the reaction well at a distance controlled by a motored stage to adjust the amplitude of magnetic field.

The 15-minute assay. For 15-minute miRNA assay, all RNA samples were protected from RNase by DEPC-treated water and $1 \% \mathrm{w} / \mathrm{v}$ SDS. DEPC treatment was essential for detection of miRNA. 1- $\mu$ l synthetic analyte miRNA was added into the $19-\mu l$ buffer containing detection-probe-conjugated AuNPs at $10^{10} / \mathrm{mL}$, and diluted to gradient concentrations from $1 \mathrm{aM}$ to $10^{7} \mathrm{fM}$. The mixture was injected into the reaction well immediately, and the SPRM continuously recorded the nanoparticle images for $15 \mathrm{~min}$. During image recording, a $+0.4 \mathrm{~V}$ voltage was applied for the first $5 \mathrm{~min}$, followed by a $-0.2 \mathrm{~V}$ voltage for $10 \mathrm{~min}$. For single base pair mismatch experiments, miR-29a and miR-29c was mixed at the concentration ratio of 1:100 before mixed with the AuNPs. The detection probe was designed to be 12 -nt complementary to miR-29a. Immunoassay via electrical manipulation follows similar workflow. For immunoassay with magnetic manipulation, $1-\mu \mathrm{l} A \beta_{1-42}$ peptide sample was added into the $19-\mu \mathrm{l}$ buffer containing $10^{9} \mathrm{NPs} / \mathrm{mL} \mathrm{MNP}$ and finally diluted to a gradient concentration of $1 \sim 10^{6} \mathrm{fg} / \mathrm{mL}$. A stack of neodymium magnets was placed at $\sim 2.5 \mathrm{~cm}$ above the reaction well at $5 \mathrm{~min}$ after the sample was injected.

Spiked tests in human serum. The human serum was collected from healthy volunteer and then centrifuged at $1500 \mathrm{rpm}$ for $10 \mathrm{~min}$. The supernatant was recovered, aliquoted, and immediately frozen. $57 \mu \mathrm{L}$ of freshly thawed human serum was mixed with $1 \% \mathrm{w} / \mathrm{v}$ SDS and proteinase $\mathrm{K}$ 
(ST533; final concentration $0.25 \mathrm{ug} / \mathrm{mL}$ ). After incubation for $30 \mathrm{~min}$ at room temperature, the EDTA was added to a final concentration of $20 \mathrm{mM}$ to inhibit the activity of proteinase $\mathrm{K} .3 \mu \mathrm{L}$ of hsa-miR-29a or $A \beta_{1-42}$ was added into pretreated-serum to the final concentrations ranging from

$1 \mathrm{fM}$ to $1 \mathrm{nM}$.

The Monte-Carlo simulation of single molecule binding kinetics. The simulation was done using a collection of 300,000 molecules at the temperature of 298 Kalvin. The temporal resolution was $0.01 \mathrm{~s}$, and the viscosity of the solution was set to be $825.9 \times 10^{-6} \mathrm{~Pa} \cdot \mathrm{S}$. The lower and higher energy barriers of molecular bound were set as $0.4373 \mathrm{~K}_{\mathrm{B}} \mathrm{T}$ and $0.0486 \mathrm{~K}_{\mathrm{B}} \mathrm{T}$ respectively.

\section{Supplementary information}

Supplementary discussion, DNA/RNA sequences and supplementary figures.

\section{Acknowledgments}

This work was funded by the National Major Scientific Research Instrument Development Program (Grant 22027807) and the National Natural Science Foundation of China (Grants 61901257 and 61805136) for financial support. The authors thank Dr. Hongchen Gu and Dr. Hong $\mathrm{Xu}$ at Shanghai Jiao Tong University for helpful discussions.

\section{Author contributions}

H.Y. and J.L. conceived the idea. Q.Z., Y.Y., X.Z., and H.Y. designed the experiments. Q.Z., X.Z. and Y.Y. carried out the experiments and analyzed the data. Y.S. J.W. and C.Z. helped with the experiments and data analysis. Q.Z., X.Z., Y.Y. J.L. and H.Y. interpreted the results and wrote the paper.

\section{Competing financial interests}

The authors declare no competing interests.

\section{References}

1. English, B. P., et al. Ever-fluctuating single enzyme molecules: Michaelis-Menten equation revisited. Nat Chem Biol 2, 87-94 (2006).

2. Velonia, K., et al. Single - enzyme kinetics of CALB - catalyzed hydrolysis. Angew Chem Intl Ed 117, 566-570 (2005).

3. Moerner, W. E., Orrit M. Illuminating single molecules in condensed matter. Science 283, 1670-1676 (1999).

4. Betzig, E., Chichester R. J. Single molecules observed by near-field scanning optical 
microscopy. Science 262, 1422-1425 (1993).

5. Venkatesan, B. M., Bashir R. Nanopore sensors for nucleic acid analysis. Nat Nanotech 6, 615-624 (2011).

6. Deamer, D., Akeson M., Branton D. Three decades of nanopore sequencing. Nat Biotechno/ 34, 518-524 (2016).

7. Mayer, K. M., Hafner J. H. Localized surface plasmon resonance sensors. Chem Rev 111, 3828-3857 (2011).

8. Zijlstra, P., Paulo P. M., Orrit M. Optical detection of single non-absorbing molecules using the surface plasmon resonance of a gold nanorod. Nat Nanotech 7, 379-382 (2012).

9. Fang, W., et al. Quantizing single-molecule surface-enhanced Raman scattering with DNA origami metamolecules. Sci Adv 5, eaau4506 (2019).

10. Nie, S., Emory S. R. Probing single molecules and single nanoparticles by surface-enhanced Raman scattering. Science 275, 1102-1106 (1997).

11. Holzmeister, P., Acuna G. P., Grohmann D., Tinnefeld P. Breaking the concentration limit of optical single-molecule detection. Chem Soc Rev 43, 1014-1028 (2014).

12. Squires, T. M., Messinger R. J., Manalis S. R. Making it stick: convection, reaction and diffusion in surface-based biosensors. Nat Biotechnol 26, 417-426 (2008).

13. Sevenler, D., Trueb J., Ünlü M. S. Beating the reaction limits of biosensor sensitivity with dynamic tracking of single binding events. Proc Natl Acad Sci USA 116, 4129-4134 (2019).

14. Kelley, S. O. What Are Clinically Relevant Levels of Cellular and Biomolecular Analytes? ACS Sens 2, 193-197 (2017).

15. Gooding, J. J., Gaus K. Single - molecule sensors: challenges and opportunities for quantitative analysis. Angew Chem Intl Ed 55, 11354-11366 (2016).

16. Wu, Y., Tilley R. D., Gooding J. J. Challenges and solutions in developing ultrasensitive biosensors. J Am Chem Soc 141, 1162-1170 (2018).

17. Wu, Y., Bennett D., Tilley R. D., Gooding J. J. How nanoparticles transform single molecule measurements into quantitative sensors. Adv Mater 32, 1904339 (2020).

18. Rissin, D. M., et al. Single-molecule enzyme-linked immunosorbent assay detects serum proteins at subfemtomolar concentrations. Nat Biotechnol 28, 595-599 (2010).

19. Wang, $H_{\text {., }}$ et al. Probing single molecule binding and free energy profile with plasmonic imaging of nanoparticles. J Am Chem Soc 141, 16071-16078 (2019).

20. Hayward, S. L., et al. Ultraspecific and amplification-free quantification of mutant DNA by single-molecule kinetic fingerprinting. J Am Chem Soc 140, 11755-11762 (2018).

21. Johnson-Buck, A., et al. Kinetic fingerprinting to identify and count single nucleic acids. Nat Biotechnol 33, 730-732 (2015).

22. Chatterjee, T., et al. Direct kinetic fingerprinting and digital counting of single protein molecules. Proc Natl Acad Sci USA 117, 22815-22822 (2020).

23. Zhang, D. Y., Chen S. X., Yin P. Optimizing the specificity of nucleic acid hybridization. Nat Chem 4, 208-214 (2012).

24. Jing, W., et al. Time-resolved digital immunoassay for rapid and sensitive quantitation of procalcitonin with plasmonic imaging. Acs Nano 13, 8609-8617 (2019).

25. Wang, Y., Jing W., Tao N., Wang H. Probing single-molecule binding event by the dynamic counting and mapping of individual nanoparticles. ACS Sens, 6, 523-529 (2020).

26. Yang, Y., et al. Quantitative amplitude and phase imaging with interferometric plasmonic 
microscopy. ACS Nano 13, 13595-13601 (2019).

27. Yang, Y., et al. Interferometric plasmonic imaging and detection of single exosomes. Proc Natl Acad Sci USA 115, 10275-10280 (2018).

28. Foote, J., Eisen H. N. Kinetic and affinity limits on antibodies produced during immune responses. Proc Natl Acad Sci USA 92, 1254 (1995).

29. Rief, M., Oesterhelt F., Heymann B., Gaub H. E. Single molecule force spectroscopy on polysaccharides by atomic force microscopy. Science 275, 1295-1297 (1997).

30. Neuman, K. C., Nagy A. Single-molecule force spectroscopy: optical tweezers, magnetic tweezers and atomic force microscopy. Nat Methods 5, 491-505 (2008).

31. Dudko, O. K., Filippov A. E., Klafter J., Urbakh M. Beyond the conventional description of dynamic force spectroscopy of adhesion bonds. Proc Natl Acad Sci USA 100, 11378 (2003).

32. Nature nanotechnologyHänggi, P., Talkner P., Borkovec M. Reaction-rate theory: fifty years after Kramers. Rev Mod Phys 62, 251 (1990).

33. Shan, X., et al. Imaging the electrocatalytic activity of single nanoparticles. Nat Nanotechnol 7, 668 (2012).

34. Shan, X., et al. Imaging local electrochemical current via surface plasmon resonance. Science 327, 1363-1366 (2010).

35. Rissin, D. M., Walt D. R. Digital readout of target binding with attomole detection limits via enzyme amplification in femtoliter arrays. J Am Chem Soc 128, 6286-6287 (2006).

36. Jing, W., et al. Gradient-Based Rapid Digital Immunoassay for High-Sensitivity Cardiac Troponin T (hs-cTnT) Detection in $1 \mu$ L Plasma. ACS Sens 6, 399-407 (2020).

37. Danilowicz, C., Greenfield D., Prentiss M. Dissociation of Ligand- Receptor Complexes Using Magnetic Tweezers. Anal Chem 77, 3023-3028 (2005).

38. Nakamura, A., et al. High performance plasma amyloid- $\beta$ biomarkers for Alzheimer's disease. Nature 554, 249-254 (2018).

39. Janelidze, S., et al. Plasma $\beta$-amyloid in Alzheimer's disease and vascular disease. Sci Rep 6, 1-11 (2016).

40. Wang, X., et al. Automated Nanoparticle Analysis in Surface Plasmon Resonance Microscopy. Anal. Chem. 93, 7399-7404 (2021). 


\section{Supplementary Files}

This is a list of supplementary files associated with this preprint. Click to download.

- SupplementaryInformation.pdf 